\title{
Polarization control of an x-ray free electron laser oscillator
}

\author{
Nanshun Huang $\odot{ }^{1,2} \mathrm{Kai}_{\mathrm{Li}}{ }^{3}$ and Haixiao Deng ${ }^{1,4, *}$ \\ ${ }^{1}$ Shanghai Institute of Applied Physics, Chinese Academy of Sciences, Shanghai 201800, China \\ ${ }^{2}$ University of Chinese Academy of Sciences, Beijing 100049, China \\ ${ }^{3}$ Department of physics, University of Chicago, Illinois 60637, USA \\ ${ }^{4}$ Shanghai Advanced Research Institute, Chinese Academy of Sciences, Shanghai 201210, China
}

(Received 27 November 2019; accepted 2 March 2020; published 13 March 2020)

\begin{abstract}
High-intensity, fully coherent x-ray pulse with a tunable polarization over a wide spectral range is of great importance to many experiments. In this paper, we propose a tapered crossed-polarized undulator configuration for an x-ray free electron laser oscillator (XFELO) to produce arbitrarily polarized $\mathrm{x}$-ray pulses in the hard $\mathrm{x}$-ray region. A numerical example utilizing the parameters of the Shanghai HighRepetition-Rate XFEL and Extreme Light Facility (SHINE) is presented to demonstrate the generation of polarization controllable, fully coherent hard x-ray pulses with $99.9 \%$ polarization degree and $20 \mathrm{kHz}$ polarization switching rate. The analysis presents that this scheme holds the possibility to be used in an energy tunable XFELO built from multiple Bragg mirrors.
\end{abstract}

DOI: 10.1103/PhysRevAccelBeams.23.030702

\section{INTRODUCTION}

Compared to the synchrotron radiation light source, the $\mathrm{x}$-ray free electron laser (XFEL) breaks new ground for $\mathrm{x}$-ray science in biology, chemistry, physics, and material sciences, for it significantly improves the peak photon brilliance, as well as the ability of controlling both the temporal duration and the spectral bandwidth of produced light pulses. The Linear Coherent Light Source (LCLS) first produced hard x-ray FEL pulses in 2009 [1]. After that, several XFELs [2-4] were constructed world widely. In recent years, a large number of complicated schemes have been developed in pursuit of, e.g., full coherence [5,6], multicolor operation $[7,8]$, ultrafast operation $[9,10]$, large bandwidth operation [11-13], and tunable polarization operation $[14,15]$.

The tunable polarization is a desirable feature of XFEL user facilities in investigating many essential properties of matter. Traditionally, in the soft $\mathrm{x}$-ray region, a circularly polarized laser is able to produce the high-resolution Fourier transform holography (FTH) image of magnetic domains [16] and to reveal the ultrafast dynamics of spin and orbital moments in solids with femtosecond $\mathrm{x}$-ray pulses [17]. In the hard x-ray region, a circularly polarized laser makes it possible to observe an inner 3-dimensional magnetic structure with a large dichroic effect for $3 d$

\footnotetext{
*denghaixiao@zjlab.org.cn
}

Published by the American Physical Society under the terms of the Creative Commons Attribution 4.0 International license. Further distribution of this work must maintain attribution to the author(s) and the published article's title, journal citation, and DOI. transition metals by employing a bulk-sensitive x-ray magnetic circular dichroism (XMCD) measurement [18-20]. Further, by using the combination of the XMCD spectroscopy and Bragg coherent diffractive imaging, one may gain the ability to study the magnetic structure and strain mapping in a single $\mathrm{x}$-ray pulse $[21,22]$. Purely $45^{\circ}$ linearly polarized hard $\mathrm{x}$-rays are also in demand for quantum electrodynamics (QED) experiments on an extreme light station [23].

However, all free electron lasers based on the planar undulator only generate linearly polarized radiation pulses in a fixed direction (horizontal or vertical). In order to obtain the ability to control the polarization of FEL pulse, two kinds of schemes were proposed and have been applied to XFEL facilities running in the external seeding and the self-amplified spontaneous emission (SASE) mode. The first method is to replace the planar undulator with an elliptically polarized undulator (EPU) [24-27], such as APPLE-type or Delta-type undulator, through which the electron beam can generate circularly polarized radiation directly. This method is mainly considered to produce the soft polarized $\mathrm{x}$-ray, for reasons that the lasing of EPU in the hard x-ray region has not been demonstrated and that the development of such undulators in the hard $\mathrm{x}$-ray region may be expensive. Besides, the XFEL equipping the EPU is unable to switch the polarization state of the produced radiation quickly, for its switching process needs a slow movement of undulator magnets. Therefore, the EPU may not be suitable for a superconducting linac based highrepetition-rate XFEL, which naturally enjoys a feature of fast polarization switching.

Another method for generating an arbitrarily polarized $\mathrm{x}$-ray is to combine two orthogonally polarized fields 


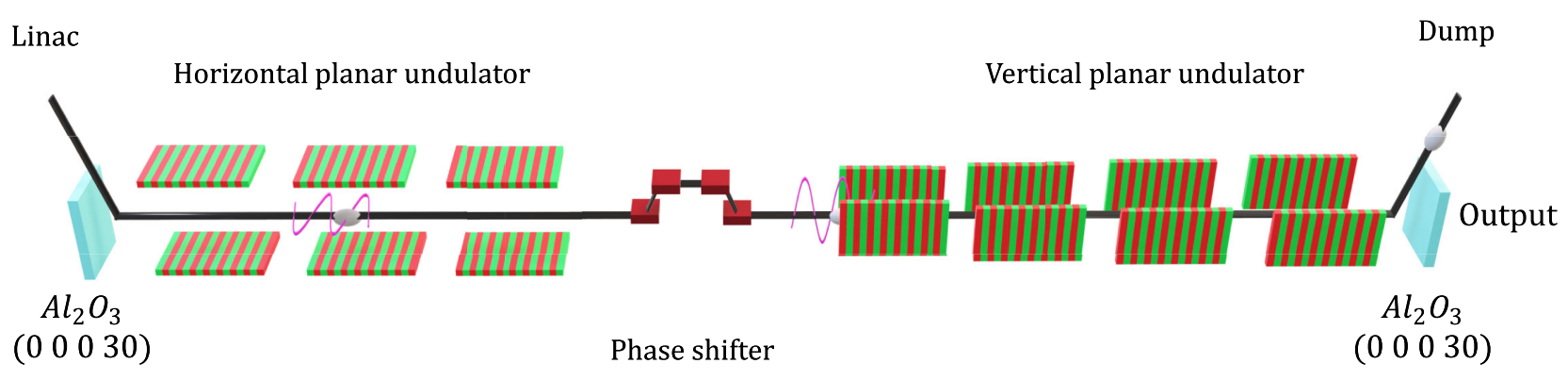

FIG. 1. A tapered crossed-polarized undulator is applied to an XFELO equipping with a pair of mirrors. The horizontally polarized radiation field is amplified in the first horizontal undulator, and the vertically polarized radiation field is amplified in the second tapered vertical undulator by a bunched electron beam. A phase shifter is placed between the undulators for controlling the polarization state. The output radiation is dumped from the downstream mirror.

produced by crossed-polarized undulators $[28,29]$. This scheme contains two planar undulators in a crossed configuration, in which two orthogonally linearly polarized fields can be generated separately. A fast phase shifter inserted between the two crossed undulator parts can control the phase difference between the two linearly polarized components. The steerable phase difference determines the final polarization state of combined radiation. The advantages of this scheme are an unrestricted lasing wavelength and a low cost. However, the polarization switching experiment using the crossed-polarized undulator [30] demonstrates that a high polarization degree requires longitudinal coherence and stability in intensity. Thus, the significant pulse-to-pulse fluctuations in intensity and spectrum of the SASE XFEL may degrade the polarization performance of generating polarized hard $\mathrm{X}$-rays by the crossed-polarized undulator [31].

With the help of superconducting MHz-rate linac and high-reflectivity crystal, the XFEL oscillator (XFELO) grows to be the candidate for producing stable, fully coherent hard x-rays $[32,33]$. The characteristics of XFELO pulses, especially the enhanced longitudinal coherence and the stability compared to the SASE XFELs, make the crossedpolarized undulator scheme greatly suitable for the polarization control of an XFELO. The numerical simulations indicate that the polarization degree can exceed $99 \%$. Furthermore, the crossed-polarized undulator is the best choice that enables the XFELO to change the polarization quickly at present. With such polarization controllable, fully coherent $\mathrm{X}$-rays, the XFELO will significantly improve the capability to generate the single-shot images of spin-resolved electronic structures [34].

In this paper, we further the study of the tapered crossedpolarized undulator scheme to generate fully coherent, arbitrarily polarized hard x-ray pulses from an XFELO. The paper is organized as follows. In Sec. II, a simple theoretical analysis is performed. In Sec. III, we conduct a numerical simulation with the parameters of the Shanghai High-Repetition-Rate XFEL and Extreme Light Facility (SHINE), the first hard $\mathrm{x}$-ray FEL facility in China.
The feasibility of using the scheme in a photon energy tunable XFELO is discussed in the following section. Finally, a summary is given in Sec. V.

\section{THEORETICAL ANALYSIS}

In the XFELO, $\mathrm{x}$-ray pulses circulate in a resonant cavity formed with Bragg reflecting mirrors. In each pass, the pulse is amplified when it overlaps with the next fresh electron beam in the undulator, and its spectrum is filtered by the Bragg reflectors. In this process, the relative growth of pulse power per pass can be represented as $G$, and the total reflectivity of mirrors with related to the cavity loss and the output coupling can be written as $R_{\text {tot }}$. When $(1+G) R_{\text {tot }}>1$, a coherent radiation pulse would gradually grow and evolves from the spontaneous radiation. After the exponential growth, the decline of $G$ occurs owing to the electron beam overmodulation caused by the high-intensity radiation. When $(1+G) R_{\text {tot }}=1$, the system eventually reaches saturation.

Figure 1 shows the diagram of the tapered crossedpolarized undulator configuration applied to an XFELO formed with two mirrors. Since the two undulator sections orient at a right-angle to each other, the two linear polarized components interact with the electron beam in the different undulator sections. For horizontally polarized field $E_{H}$, it is always amplified by a fresh electron beam. So that the amplification model of $E_{H}$ can be simplified as an ordinary XFELO using only the first horizontal planar undulator section. Generally, the saturation electron-to-light energy conversion efficiency of an XFELO can be simply evaluated by $\eta_{H}=1 / 2 N_{H}$ [35,36]. $N_{H}$ is the number of undulator periods, which is defined by the undulator length $L_{H}$. This formula shows that the saturation energy of $E_{H}$ is negatively proportional to $L_{H}$. Meanwhile, to guarantee the cavity stability and to grow the radiation field, a larger $L_{H}$ is preferable. Thus, the optimization of the first undulator section is to balance the cavity stability and the output power by selecting the proper number of the undulator segments. 
For vertically polarized field $E_{V}$, its amplification process is inevitably influenced by $E_{H}$ through the dumped electron beam. At saturation, the dumped electron beam has two main characters: (1) a large bunching factor; (2) a large energy spread. These characters are due to the energy modulation and the density modulation of the FEL amplification process with the high-intensity $E_{H}$ in the first undulator section. Therefore, a postsaturation undulator taper technique is utilized to amplify $E_{V}$ by a bunched electron beam with a large energy spread.

In the Kroll-Morton-Rosenbluth (KMR) model of tapered FEL [37], the resonant phase $\psi_{r}$ is given by

$$
\frac{d \gamma_{r}(z)}{d z}=-\frac{e K[J J] E_{V}}{2 \gamma_{r}(z) m c^{2}} \sin \psi_{r}
$$

Then, the energy extraction efficiency of vertical direction can be written as:

$\eta_{V} \approx \frac{e}{2 m_{e} c^{2} \gamma_{0}^{2}} \int_{0}^{L_{V}} K_{V}(z)[J J] f_{\Lambda}(z) E_{V}(z) \sin \psi_{r}(z) d z$

where $f_{\Lambda}$ is the fraction of trapped electrons, $\gamma_{0}$ is the electron energy, $\gamma_{r}$ is the resonant energy, $K_{V}$ is the undulator parameter, $E_{V}$ is the field amplitude of the vertically polarized radiation, $c$ is the light speed, and $m_{e}$ is the electron mass [35]. Here, the formula is based on an assumption that the electron energy is constant. It is a good approximation in a low-gain FEL [38]. The tapered profile $K_{V}(z)$ needs to be optimized to match energy extraction efficiency in both directions. Thus, the optimization of the second undulator section is to decrease the undulator length $L_{V}$ and to make the intensity of $E_{V}$ close to that of $E_{H}$.

Unlike the single-pass FEL, the phase of XFELO pulse changes with the round trip, and the field $E_{V}$ is not generated from noise but amplified in the second undulator. According to the FEL theory, the amplification of $E_{V}$ requires the phase of the field to match that of electron beam bunching [39-41]. If it does not match, the trapped fraction $f_{\Lambda}$ would be small, which translates into a low efficiency of the vertical direction. However, the phase of electron beam bunching relates to the phase of $E_{H}$. The combined result is that the amplification of $E_{V}$ requires the phase change of $E_{V}$ to match that of $E_{H}$ in one round trip. Thus, the optimization of the taper profile of the second undulator must consider to match it. While the taper profile is optimized by a genetic algorithm, three aspects should be considered. These aspects are (1) the pulse energy at the end of the undulator; (2) the integral pulse energy over the undulator; (3) the phase change of $E_{V}$ to match that of $E_{H}$ in one round trip.

According to the superposition principle, the total field is given by

$$
E_{T}=E_{H}+E_{V} e^{i \phi}
$$

Here, the relative phase difference $\phi$ determining the final polarization state can be separated in two parts as $\phi=\phi_{0}+\delta \phi$. The first part, $\phi_{0}$, is the generic phase difference between the horizontal and vertical components. It is different from the single-pass FEL that $\phi_{0}$ is always changing until $E_{H}$ and $E_{V}$ reach steady-state in the XFELO. The second part, $\delta \phi$, is an additional phase modulation, which can be varied by tuning the phase shifter inserted between the two undulator sections.

In this case, the polarization properties can be studied by using the formulas of Stokes parameters which are defined as [42]:

$$
\begin{aligned}
& S_{0}=\left|E_{H}\right|^{2}+\left|E_{V}\right|^{2}=I_{H}+I_{V}, \\
& S_{1}=\left|E_{H}\right|^{2}-\left|E_{V}\right|^{2}=I_{H}-I_{V}, \\
& S_{2}=\Re\left(E_{H} E_{V}\right)=I_{45^{\circ}}-I_{135^{\circ},} \\
& S_{3}=\Im\left(E_{H} E_{V}\right)=I_{R}-I_{L} .
\end{aligned}
$$

In the equations above, $I_{H}, I_{V}, I_{45^{\circ}}$, and $I_{135^{\circ}}$ are the intensity of a linearly polarized component over the indicated direction, $I_{R}$ and $I_{L}$ are the intensity of right-hand and left-hand circularly polarized components respectively.

Using the Stokes parameters in Eq. (4), the linearly and circularly polarized factors of the radiation are

$$
\begin{aligned}
& P_{l}=\frac{S_{2}}{S_{0}}, \\
& P_{c}=\frac{S_{3}}{S_{0}} .
\end{aligned}
$$

Then, the polarization states of light can be described as, i.e., $P_{c}=1$ for right-hand circular polarization, $P_{c}=-1$ for left-hand circular polarization, $P_{l}=1$ for $+45^{\circ}$ linearly polarization, and $P_{l}=-1$ for $135^{\circ}$ linear polarization. Therefore, the polarization degree can be expressed as $\left|P_{c}\right|$ and $\left|P_{l}\right|$ for circular and linear polarization, respectively. In addition, we use the on-axis radiation intensity and phase to calculate the degree of polarization.

\section{AN EXAMPLE DESIGN FOR SHINE}

In this section, we simulate the crossed-polarized undulator scheme on an XFELO operating at the parameters of SHINE. As a superconducting accelerator-based XFEL, the electron beam will have a repetition rate of $1 \mathrm{MHz}$, an energy of $8 \mathrm{GeV}$, and a $100 \mathrm{pC}$ charge compressed to the peak current of $1000 \mathrm{~A}$. Here, we note that the peak current is lower and the bunch length is longer than the SASE mode. Because the short pulse would generate a larger bandwidth, which results in a lower integral reflectivity over the spectrum and a larger coupling output in the XFELO. So that 1000 A peak current with a longer bunch 
TABLE I. The main parameters of XFELO operation for SHINE.

\begin{tabular}{lc}
\hline \hline Parameter & Value \\
\hline Beam energy & $8 \mathrm{GeV}$ \\
Relative energy spread & $0.01 \%$ \\
Normalized emittance & $0.4 \mathrm{~mm} \cdot \mathrm{mrad}$ \\
Peak current & $1000 \mathrm{~A}$ \\
Undulator period length & $16 \mathrm{~mm}$ \\
Undulator segment length & $4 \mathrm{~m}$ \\
Photon energy & $14.3 \mathrm{keV}$ \\
Mirror material & Sapphire \\
Bragg mirror reflectivity, $R_{\mathrm{tot}}$ & $80 \%$ \\
Darwin width & $13 \mathrm{meV}$ \\
\hline \hline
\end{tabular}

length is selected. Additionally, SHINE will equip gapvariable undulators each $4 \mathrm{~m}$ long with a period length of $16 \mathrm{~mm}$. The phase shifter will be inserted at the break section between two undulator segments. Additional parameters are listed in Table I.

The optical cavity is built from two sapphire $(0,0,0,30)$ crystal mirrors. The length of the cavity reaches $150 \mathrm{~m}$ to match the repetition rate of the electron beam. For the whole cavity, the total reflectivity $R_{\text {tot }}$ at the Bragg energy of $14.3 \mathrm{keV}$ reaches $80 \%$ (coupling output is $17 \%$ ). Meanwhile, the high-reflectivity spectral bandwidth of the Bragg reflecting mirror is nearly $13 \mathrm{meV}$, beyond which it filters out frequency components.

In the cavity, nine undulator segments are employed. Three of the undulator segments are horizontal, in order for the horizontally polarized radiation $E_{H}$ to grow. The other six segments are vertically placed. Since the intensity of $E_{V}$ is required to be the same as that of $E_{H}$, the undulator parameter $K_{V}$ of those six segments is carefully optimized by an evolution algorithm. Figure 2 presents a satisfactory

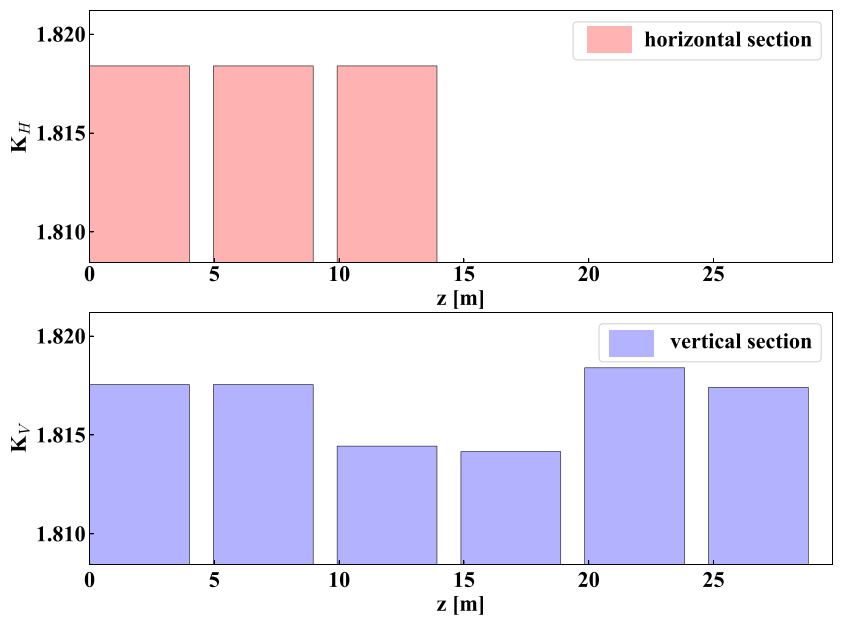

FIG. 2. The undulator parameters in the first undulator section. The optimized taper profile of the second undulator section. Each parameter of the six undulators is optimized to match the pulse energy produced by the first undulator section. taper profile. In this case, the undulator parameter is not exactly tapered. This is mainly due to the requirement that the phase change needs to be matched in two directions. The last segment can be utilized to precisely control the intensity of $E_{V}$. In addition, a stairlike taper is better for evaluating the real FEL performance than a continuous one.

The simulations are conducted by using the combination of a time-dependent FEL code GENESIS [43], a field propagation simulation code OPC [44], and a Bragg reflection simulation code BRIGHT [45]. Here, we simulate the FEL lasing process in the two undulator sections independently. The electron beam distribution file is dumped at the end of the first undulator section and is then loaded as an input in the second undulator section.

Figures 3 and 4 show the simulation results including the pulse energy growth, the longitudinal characteristics, and the transverse mode. The left plot of Fig. 3 shows the evolution of the pulse energy in both horizontal (red line) and vertical (blue line) direction, while the corresponding phase difference $\phi$ is presented as a purple line. After a short period of struggling as the initial shot noise, the laser energy increases as it goes through the undulator again and again. Reached saturation, the output pulse energy stays $162 \mu \mathrm{J}$ at each polarized direction and the total power reaches $325 \mu \mathrm{J}$ corresponding to $1.4 \times 10^{11}$ photons. Thanks to the phase of the field and its intensity are coupled together, $\phi$ varies with pulse energy. The variation of $\phi$ has three stages: irregular variation, regular variation, and steady-state with a small oscillation. Before the exponential growth, the bunching factor is not large enough to influence the phase of $E_{V}$. So, $E_{V}$ and $E_{H}$ grow independently, and $\phi$ varies irregularly. As the phase of $E_{V}$ leads by the bunching phase, regular variation occurs. After the pulse energy saturated, $\phi$ reaches a steady-state. At the steady-state, $\phi$ has a small oscillation resulting from the small intensity fluctuation of $E_{H}$, which is an XFELO nature.

The central plot of Fig. 3 presents the spectrum of the radiation pulse. The right plot of Fig. 3 shows a typical saturation power profile with a peak power of $1.2 \mathrm{GW}$. The $\mathrm{x}$-ray pulse is a Gaussian-like single peak in both time and spectrum. The FWHM spectral width is about $25 \mathrm{meV}$, while the temporal width fixed by the current profile is about 114 fs. This corresponds to a bandwidth-temporal product of 0.68 , approaching the Fourier limit of 0.44 for a Gaussian pulse profile.

It is the most critical consideration in perfect polarization control of the crossed-polarized undulator that the radiation pulses from the two undulator sets should be as identical as possible. Fortunately, the negligible slippage effect of the low gain device and the narrow crystal spectral filtering width give advantages to this goal. The negligible slippage effect ensures the generation of two fields with a nearly identical profile. The brilliant longitudinal coherence guarantees the coherent superposition of the two components. 

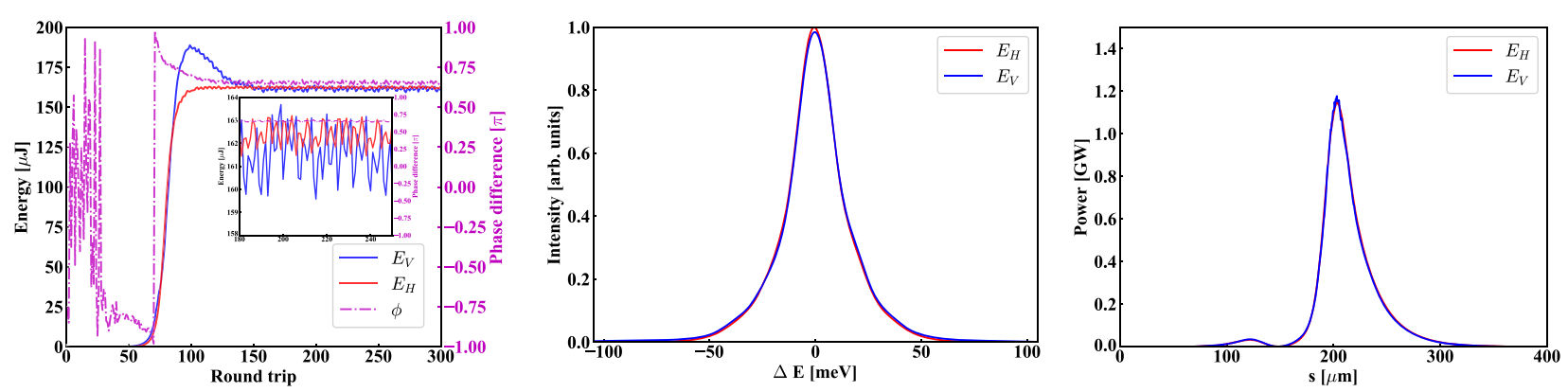

FIG. 3. The pulse energy evaluation (left), the spectrum of the radiation (center), and the temporal power profile (right) are shown. The phase difference (purple line) between the two linearly polarized components is presented in the left plot. The total energy is about $325 \mu \mathrm{J}$, the spectrum has an FWHM width of $25 \mathrm{meV}$, and the pulse has a peak power of $1.2 \mathrm{GW}$.
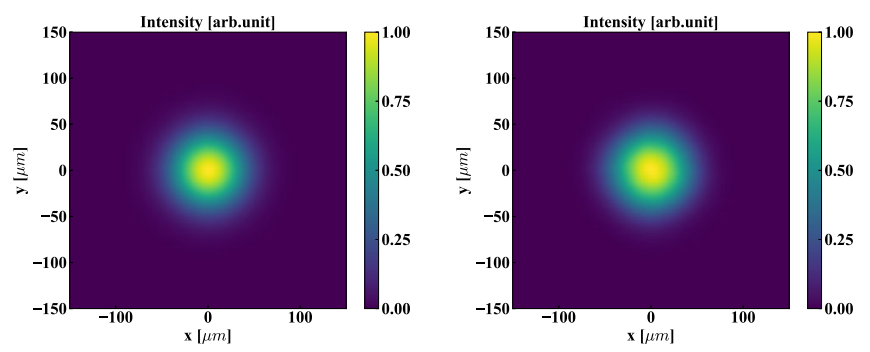

FIG. 4. The transverse radiation profiles of $E_{H}$ (left column) and $E_{V}$ (right column) are shown. It demonstrates that the output has a Gaussian mode.

Thus, the combined radiation is expected to have an excellent polarization performance.

Also, the transverse radiation profiles are reported in Fig. 4. The radiation at each direction holds a nearly identical transverse size. This is because the XFELO transverse mode is determined by an additional focusing optical element, which traps the radiation and controls the radiation size. The nearly identical transverse mode would help to avoid the reduction of polarization when the radiation propagates in free space between the source and the experimental place.

\section{A. Right-hand and left-hand circular polarization}

Benefitted from the high stability of the XFELO, the output polarized $\mathrm{x}$-ray pulse is expected to be of high robustness. The circular polarization performance are presented in Fig. 5 in both left-hand (red) and right-hand (blue) polarized state. The statistical result is obtained over 250 shots with different initial random seeds in the GENESIS simulation. The average degree of circular polarization exceeds $99.9 \%$ for both polarized state, while the RMS fluctuation level is $0.1 \%$. Compared to the SASE XFEL, the XFELO using the crossed undulator can produce a very high polarization degree in both the left-hand and the righthand circular state. The simulation results confirm that the performance of the crossed-polarized undulator highly relies on the coherence of two components.
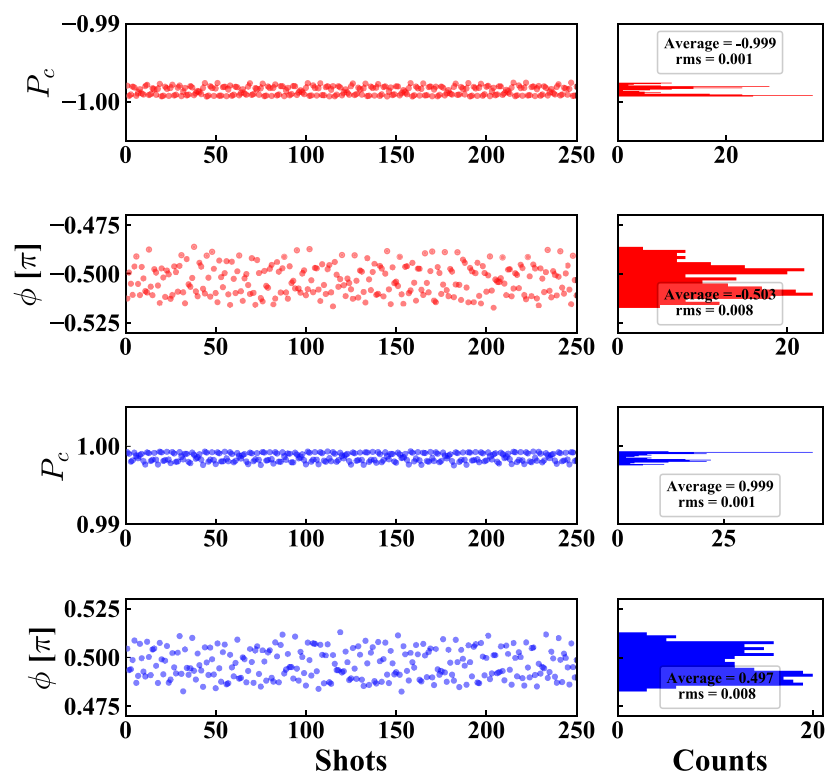

FIG. 5. $\quad P_{c}$ and phase difference $\phi$ are shown when the phase shifter is tuned to left-hand circular and right-hand circular polarization. The histograms show the distribution of the data. The average polarization degree $\left|P_{c}\right|$ approach $99.9 \%$ with $0.1 \%$ RMS fluctuation. The histograms also show that the phase difference $\phi$ is stable.

Such is an XFELO nature that its output has a small fluctuation. However, the polarization degree fluctuation is enlarged by the strong coupling between $E_{H}$ and $E_{V}$. This is because $E_{V}$ is more sensitive to the electron beam quality. So that the intensity and phase fluctuations of $E_{V}$ are larger than those of $E_{H}$, and then the polarization degree fluctuation of the combined radiation is enlarged. Even so, the final polarization fluctuation is expected to be much smaller than that of SASE XFEL.

\section{B. $45^{\circ}$ linear and $135^{\circ}$ linear polarization}

We calculate the polarization properties of linearly polarized radiation, again by changing the phase shifter. Figure 6 shows the calculated results with related to the $45^{\circ}$ 

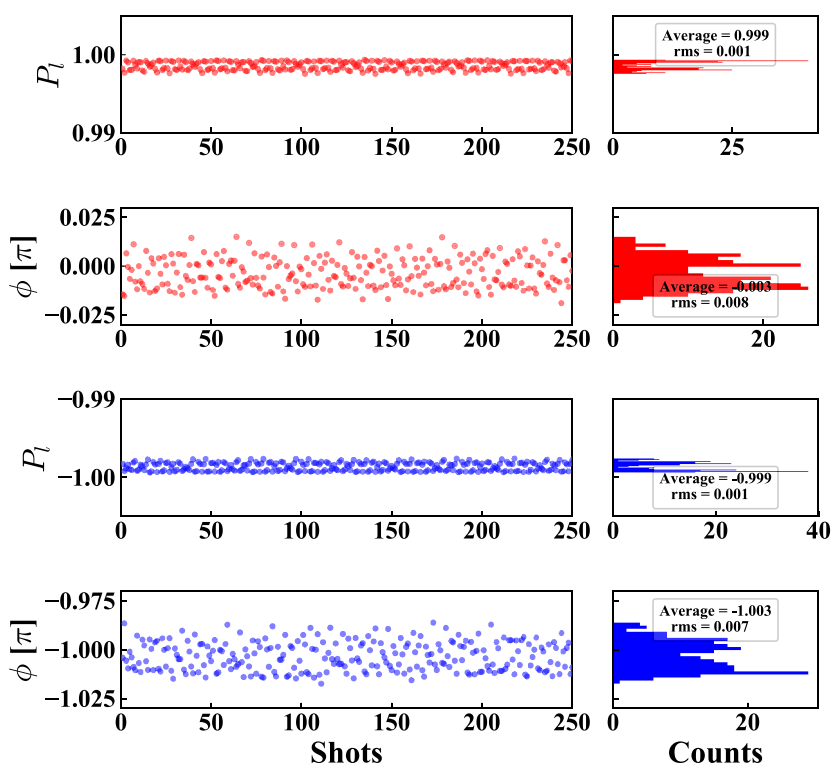

FIG. 6. $\quad P_{l}$ and phase difference $\phi$ are shown when the phase shifter is tuned to $45^{\circ}$ linear and $135^{\circ}$ linear polarization. The histograms show the distribution of the data. The average polarization degree $\left|P_{l}\right|$ approach $99.9 \%$ with $0.1 \%$ RMS fluctuation.

linearly (red) and $135^{\circ}$ linearly (blue) polarized state. The averaged value for each quantity is obtained over 250 shots. The two linear polarization states reach the same level of polarization degree $(99.9 \%)$ and RMS fluctuation $(0.1 \%)$, which is similar to the circular polarization seen in Fig. 5.

In vacuum birefringence experiments, a high intensity linearly polarized x-ray is in demand and its polarization purity needs to be above $10^{-6}$ [23]. Besides, the polarization of the x-ray pulses should be initially oriented at an angle of $45^{\circ}$ in the experiment, for the $100 \mathrm{PW}$ laser cannot simply change its polarization. Currently, since the SASE line at SHINE can not generate the $45^{\circ}$ linearly polarized $\mathrm{x}$-ray with required purity, it is planned to be generated by multiple polarizers, e.g., channel-cut crystal. Even if the SASE line generates it, the shot-to-shot intensity fluctuation will be nearly $100 \%$, while the monochromator is used to increase polarization purity. In this case, the XFELO equipping with the crossed-polarized undulator can significantly improve the spectral brilliance and stability, which can greatly improve the experimental results and reduce the experimental time.

\section{Polarization switching}

To demonstrate the performance of polarization switching, the phase shifter is adjusted, which changes the polarization from $45^{\circ}$ linear to left-handed circular, then to right-handed circular, finally to $135^{\circ}$ linear. The calculated $P_{c}$ and $P_{l}$ are given in Fig. 7. As $E_{H}$ stays saturation, the electron beam is always bunched in the second undulator. The bunched electron beam can reduce the saturation time of $E_{V}$ significantly. In this condition, the

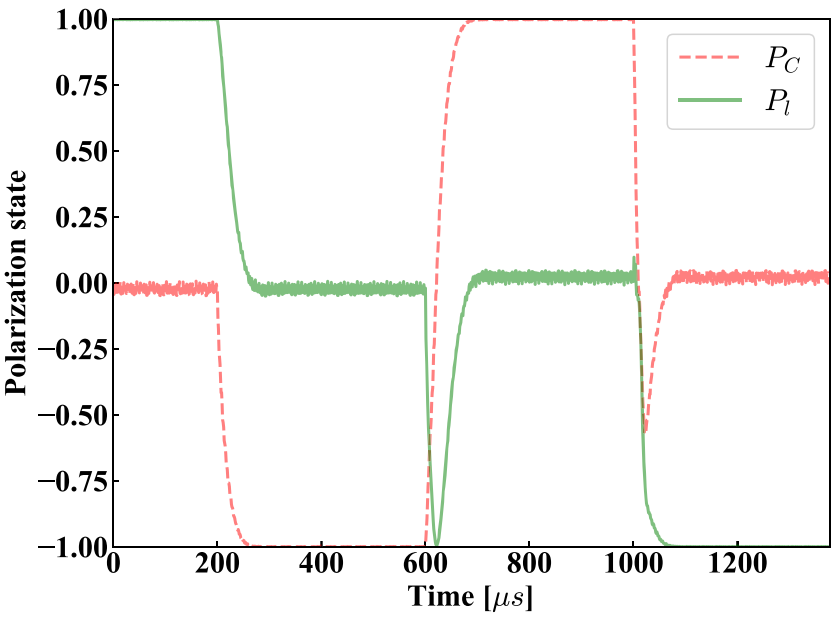

FIG. 7. The evolution of the polarization state. $P_{c}$ displays by red dash line and $P_{l}$ displays by the green line. After changing the phase shifter, phase difference becomes stable within about 50 passes for different polarized states.

switching time can be approximated as the exponential growth time of $E_{V}$. In our case, the polarization switching is accomplished in about 50 passes, 50 microseconds for $1 \mathrm{MHz}$ repetition rate, seen in Fig. 7. This switching rate is four orders of magnitude faster than those requiring the mechanical adjustments of undulator magnets.

In the single-pass high-gain XFEL, the phase of $E_{V}$ can be changed immediately in the next shot, once the phase shifter is changed. However, it becomes different in the XFELO. The vertical field $E_{V}$ will be regenerated with the new phase in the next tens round trip. This is an obvious disadvantage of the XFELO in comparison to the singlepass XFEL. A possible way to accelerate the polarization switching is to increase the single-pass gain. As long as the single-pass gain is high enough, the regeneration of $E_{V}$ can be accomplished within a few round trips. Nevertheless, this method may decrease the saturation power according to the FEL theory.

The phase shifter changes the longitudinal phase of the electron beam typically by inducing an additional traveled distance of the electron beam. However, the delayed distance of the phase shifter is so small that its effects on the bunching factor could be negligible. Generally, a conventional permanent magnet phase shifter cannot work at such high switching rate. For the high-repetition-rate XFEL, such a switching process can use an electromagnetic phase shifter on tens $\mathrm{kHz}$ or a phase shifter like the fast kicker on faster repetition rates. Additionally, the reliability of the phase shifter is a critical factor in polarization switching, which should be optimized in design.

\section{DISCUSSION FOR PHOTON ENERGY TUNABLE XFELO}

The resonant cavity for the energy tunable XFELO consists of four crystal mirrors $[35,46]$. The photon energy 


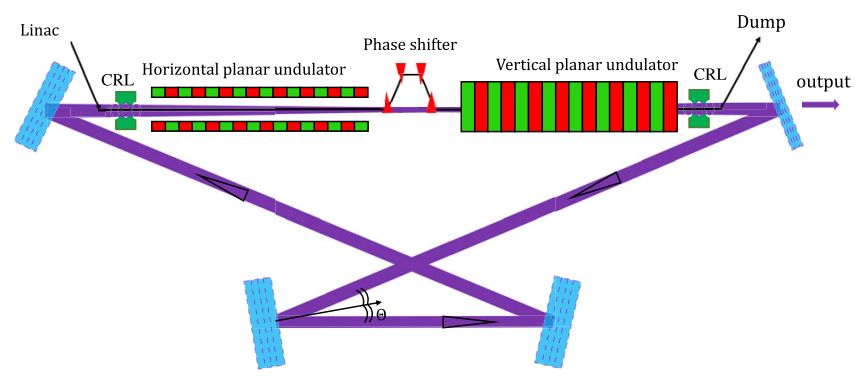

FIG. 8. A schematic configuration for the tunable cavity. Four Bragg mirrors are used. When changing the photon energy, the incidence angle of all four mirror must change simultaneously.

is tuned by adjusting the angle of $\mathrm{x}$-ray incidence on four mirrors, seen in Fig. 8. The range of tunable energy is

$$
E_{B} \leq E \leq E_{B} / \cos \Theta_{\max }
$$

where $\Theta=\pi / 2-\theta_{B}, E_{B}$ is Bragg energy and $\theta_{B}$ is Bragg angle, the angle between the incident optical axis and the crystal plane, shown in Fig. 8, and $\Theta_{\max }$ is the maximum tunable angle. Generally, $\Theta_{\max }$ is less than $20^{\circ}$, so that the tuning energy range at $14.3 \mathrm{keV}$ is about $917 \mathrm{eV}$. Using the crossed-polarized undulator in the tunable cavity, one should account for the impact that the radiation at different polarized directions has a nonequal phase shift and a nonequal reflectivity at non-normal incidence.

As mentioned above, the Bragg mirror would introduce an extra phase difference between the horizontally polarized and the vertically polarized components. Since the angular divergence of the XFELO beam is sufficiently smaller than the Darwin width, we only consider the effect over the relative energy $\Delta E$. Figure 9 shows the reflectivity

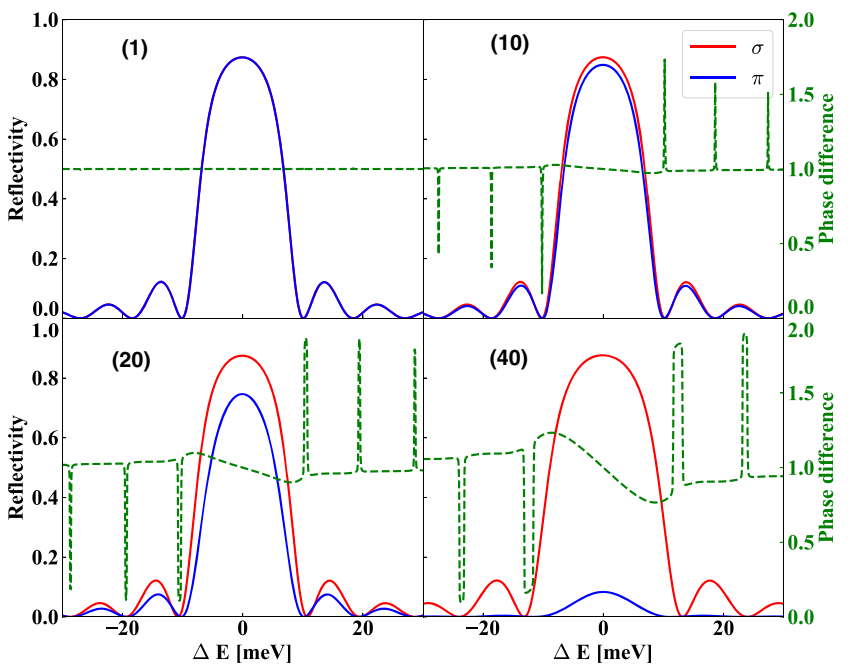

FIG. 9. The rocking curves with the additional phase difference between the horizontally polarized and the vertically polarized components are shown. The calculated results are based on the dynamical theory of $\mathrm{x}$-ray diffraction when $\Theta$ is $1^{\circ}, 10^{\circ}, 20^{\circ}$, and $40^{\circ}$. on different polarization directions and the corresponding phase difference (green line), when $\Theta$ is $1^{\circ}, 10^{\circ}, 20^{\circ}$, and $40^{\circ}$. The reflectivity is calculated on diamond ( $\left.\begin{array}{lll}3 & 3 & 7\end{array}\right)$ reflection where $E_{H}$ is $14.225 \mathrm{keV}$. As the XFELO pulse is not purely monochromatic, the phase shift may be significantly different for large $\Delta E$ at the edge of the Darwin width, seen in Fig. 9. However, there is a limited effect because the spectral width of the radiation pulse can be narrower than the Darwin width and the intensity-weighted average of the phase difference is small enough. Thus, the high polarization degree can be maintained after the Bragg reflection. Further, the crystal mirrors in a dispersive arrangement, e.g., fully symmetric $(+--+)$ setting, may cancel the phase variation and transfer the circularly polarized radiation in the cavity $[47,48]$.

The top plot of Fig. 10 shows the reflectivity on different polarization at $\Theta$ in range from $1^{\circ}$ to $40^{\circ}$. The reflectivity difference between horizontally polarized and vertically polarized components is small when $\Theta$ is small. While $\Theta$ is smaller than $20^{\circ}$, the ratio in the Bragg reflectivity of the two polarizations can exceed 0.85 , which is acceptable. The bottom plot of Fig. 10 displays the reflectivity ratio of two components with different crystal thickness. A $200 \mu \mathrm{m}$
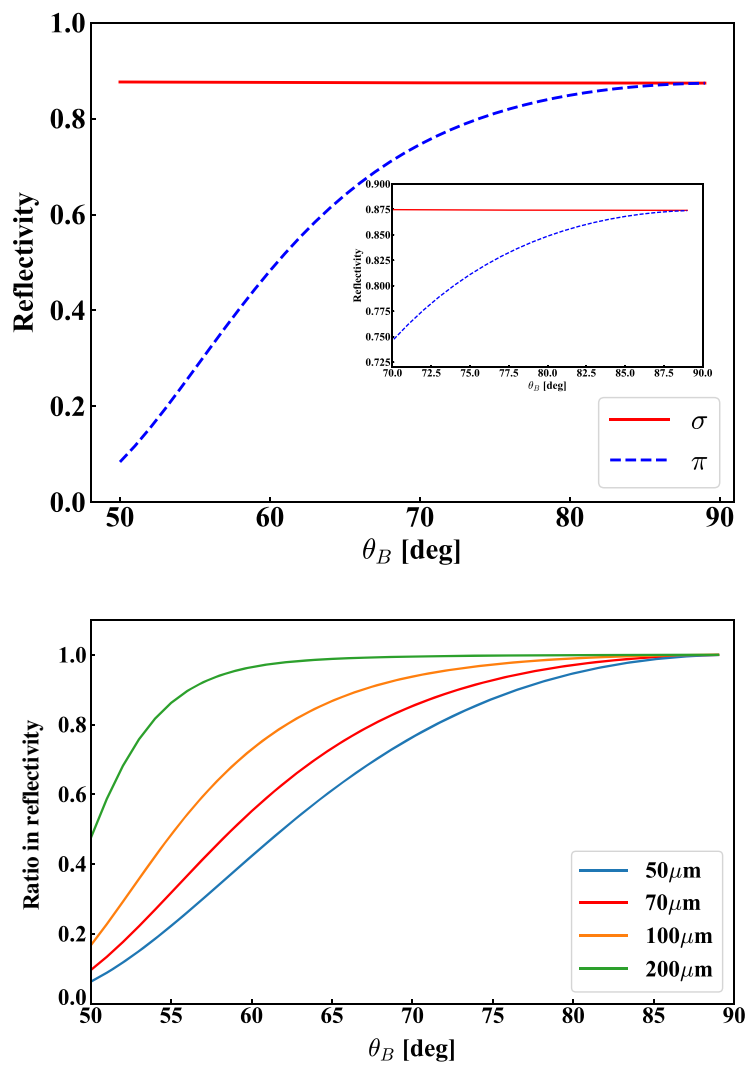

FIG. 10. The top plot shows the reflectivity in different polarized directions at different $\theta_{B}$. The bottom plot shows the ratio of reflectivity in the two polarizations with different crystal thickness. The ratio defines as the reflectivity in $\pi$ polarization divides by that in $\sigma$ polarization. 
thick crystal will generate nearly identical reflectivity when $\theta_{B}$ is larger than $60^{\circ}$. However, when the mirrors are thick crystals, the output coupling would be a problem. New methods to dump the radiation out the cavity need to be developed, e.g., an additional thin crystal is inserted to extract a part of the radiation [49]. In this situation, the intensity error resulting from the nonequal reflectivity can be compensated to a negligible level by tuning the gain of each polarized direction, e.g., by tuning the taper. Thus, the nonequal reflectivity effect may not prevent the scheme from working, but limit the tunable Bragg angle and the saturation power.

Additionally, the Bragg crystal mirror would produce an extra time delay attributed to the time for the radiation to penetrate crystal [46]. The penetration distance of the Bragg reflection relates to the crystal extinction length, which can be expressed as

$$
L_{H}^{\mathrm{ext}}=\frac{\lambda_{H}}{2 \pi P\left|\chi_{H}\right|}
$$

where $\lambda_{H}$ is the wavelength of the radiation, $\chi_{H}$ is the Fourier coefficient of crystal electric susceptibility. $P$ is polarization factor, $P=1$ for the $\sigma$-polarization component, and $P=\cos 2 \theta_{B}$ for the $\pi$-polarization component [50]. Thus, the ratio in the extinction length of the two polarizations is $\left|\cos 2 \theta_{B}\right|$. When $\theta_{B}$ is close to $45^{\circ}$, the ratio would be extremely small. For a short pulse, it may become a fatal disease.

In symmetric scattering geometry, the variation of the extinction length from different reflections is shown in Fig. 11. Generally, $L_{H}^{\text {ext }} \approx 1-50 \mu \mathrm{m}$ and it increases with the Bragg energy $E_{B}$. While $\theta_{B}$ is $70^{\circ},\left|\cos 2 \theta_{B}\right|=$ $\left|\cos 140^{\circ}\right|=0.77$ and the difference of delayed distance is $0.92-46 \mu \mathrm{m}$ for four Bragg mirrors. In this condition, the bunch length of the electron beam should be a picosecond level to mitigate the impact of different penetration distance. As described above, for high energy photons and

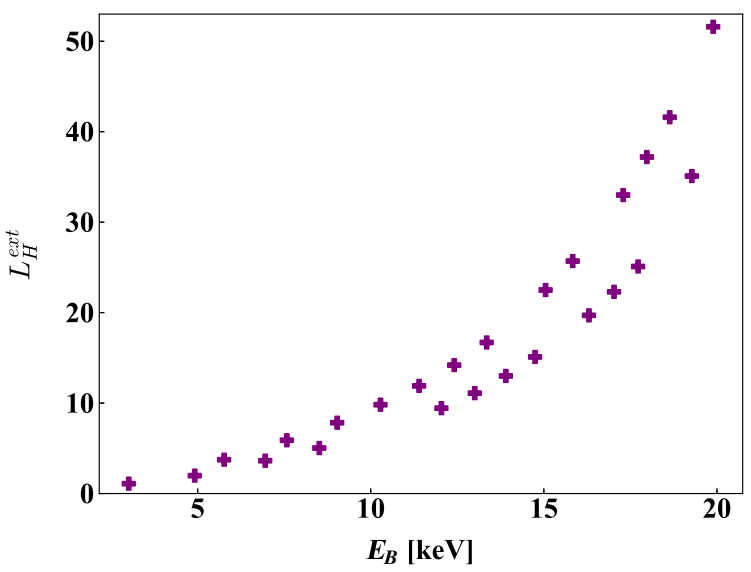

FIG. 11. Calculated extinction length for $\mathrm{x}$-rays in Bragg backscattering from different reflections. small Bragg angles, one should increase the bunch length to reduce the influence of the different time delays.

\section{CONCLUSION}

In this paper, we exploit the tapered crossed-polarized undulator scheme for the XFELO device to achieve the ultimate performance of polarization controlling, high polarization degree generating, and fast switching. In this scheme, the undulator line is divided into two sections, and the taper profile of the second section should be optimized to match the radiation power generated in the first section. The theoretical analysis of the energy extraction efficiency of two directions is conducted, and a simple strategy to optimized the taper profile is presented.

We present an illustration of the scheme for SHINE. The proposed scheme can generate fully coherent $\mathrm{x}$-ray pulses with $99.9 \%$ polarization degree and $0.1 \%$ RMS fluctuation. The polarization state can be varied arbitrarily by switching the phase shifter inserted between the two undulator sections, according to the needs of users. The main advantage of this scheme appears to be a high polarization degree in hard $\mathrm{x}$-ray region with fast polarization switching, up to dozens-kHz level, which is four orders of magnitude faster than those requiring mechanical adjustments. The possibility of using this scheme in a tunable cavity is discussed. The discussion demonstrates that this scheme can be used in the cavity tunable XFELO but limits the adjustable energy range, the Bragg angle, and the bunch length of the electron beam. The proposed method provides a powerful tool for the polarization experiments of $\mathrm{x}$-ray FEL oscillator facilities.

\section{ACKNOWLEDGMENTS}

The authors are grateful to J. Yan for helpful discussions and useful comments. This work was partially supported by the National Key Research and Development Program of China (Grants No. 2018YFE0103100, No. 2016YFA0401900) and the National Natural Science Foundation of China (Grants No. 11935020, No. 11775293).

[1] P. Emma, R. Akre, J. Arthur et al., First lasing and operation of an ångstrom-wavelength free-electron laser, Nat. Photonics 4, 641 (2010).

[2] T. Ishikawa, H. Aoyagi, T. Asaka, Y. Asano, N. Azumi, T. Bizen, H. Ego, K. Fukami, T. Fukui, Y. Furukawa et al., A compact X-ray free-electron laser emitting in the sub-ångström region, Nat. Photonics 6, 540 (2012).

[3] H.S. Kang et al., Hard X-ray free-electron laser with femtosecond-scale timing jitter, Nat. Photonics 11, 708 (2017).

[4] C. Milne, T. Schietinger, M. Aiba, A. Alarcon, J. Alex, A. Anghel, V. Arsov, C. Beard, P. Beaud, S. Bettoni et al., SwissFEL: The Swiss X-ray Free Electron Laser, Appl. Sci. 7, 720 (2017). 
[5] J. Amann, W. Berg, V. Blank, F.-J. Decker, Y. Ding, P. Emma, Y. Feng, J. Frisch, D. Fritz, J. Hastings et al., Demonstration of self-seeding in a hard-X-ray freeelectron laser, Nat. Photonics 6, 693 (2012).

[6] C. Feng and H. X. Deng, Review of fully coherent freeelectron lasers, Nucl. Sci. Tech. 29, 160 (2018).

[7] A. Marinelli, D. Ratner, A. A. Lutman, J. Turner, J. Welch, F. J. Decker, H. Loos, C. Behrens, S. Gilevich, A. A. Miahnahri, S. Vetter, T. J. Maxwell, Y. Ding, R. Coffee, S. Wakatsuki, and Z. Huang, High-intensity double-pulse X-ray free-electron laser, Nat. Commun. 6, 6369 (2015).

[8] J. Qiang and J. Wu, in Proceedings of the 32nd Free Electron Laser Conference, Malmöö, Sweden (Max-lab, Sweden, 2010), p. 290.

[9] A. A. Lutman, T. J. Maxwell, J. P. MacArthur, M. W. Guetg, N. Berrah, R. N. Coffee, Y. Ding, Z. Huang, A. Marinelli, S. Moeller, and J. C. Zemella, Fresh-slice multicolour X-ray free-electron lasers, Nat. Photonics 10, 745 (2016).

[10] Z. Zhang, J. Duris, J. P. Macarthur, Z. Huang, and A. Marinelli, Double chirp-taper x-ray free-electron laser for attosecond pump-probe experiments, Phys. Rev. Accel. Beams 22, 050701 (2019).

[11] J. Yan and H. Deng, Generation of large-bandwidth X-ray free electron laser with evolutionary many-objective optimization algorithm, Phys. Rev. Accel. Beams 22, 020703 (2019).

[12] B. Deng, J. Yan, Q. Zhang, Y. Sang, and H. Deng, Proposal of X-ray absorption spectroscopy and magnetic circular dichroism using broadband free-electron lasers, J. Synchrotron Radiat. 26, 1 (2019).

[13] J. Yan and H. Deng, Multi-beam-energy operation for the continuous-wave x-ray free electron laser, Phys. Rev. Accel. Beams 22, 090701 (2019).

[14] E. Ferrari, E. Roussel, J. Buck, C. Callegari, R. Cucini, G. De Ninno, B. Diviacco, D. Gauthier, L. Giannessi, L. Glaser, G. Hartmann, G. Penco, F. Scholz, J. Seltmann, I. Shevchuk, J. Viefhaus, M. Zangrando, and E. M. Allaria, Free electron laser polarization control with interfering crossed polarized fields, Phys. Rev. Accel. Beams 22, 080701 (2019).

[15] A. A. Lutman et al., Polarization control in an X-ray freeelectron laser, Nat. Photonics 10, 468 (2016).

[16] S. Eisebitt, J. Lüning, W. F. Schlotter, M. Lörgen, O. Hellwig, W. Eberhardt, and J. Stöhr, Lensless imaging of magnetic nanostructures by X-ray spectro-holography, Nature (London) 432, 885 (2004).

[17] C. Boeglin, E. Beaurepaire, V. Halté, V. López-Flores, C. Stamm, N. Pontius, H. A. Dürr, and J. Y. Bigot, Distinguishing the ultrafast dynamics of spin and orbital moments in solids, Nature (London) 465, 458 (2010).

[18] T. Inami, Magnetic Circular Dichroism in X-Ray Emission from Ferromagnets, Phys. Rev. Lett. 119, 137203 (2017).

[19] K. Sato, Y. Ueji, K. Okitsu, T. Matsushita, J. Saito, T. Takayama, and Y. Amemiya, Hard X-ray Magnetic Linear Dichroism Imaging, Trans. Magn. Soc. Jpn. 2, 238 (2002).

[20] H. Fujiwara, S. Naimen, A. Higashiya, Y. Kanai, H. Yomosa, K. Yamagami, T. Kiss, T. Kadono, S. Imada, A. Yamasaki, K. Takase, S. Otsuka, T. Shimizu, S. Shingubara, S. Suga, M. Yabashi, K. Tamasaku, T. Ishikawa, and A. Sekiyama, Polarized hard X-ray photoemission system with micro-positioning technique for probing ground-state symmetry of strongly correlated materials, J. Synchrotron Radiat. 23, 735 (2016).

[21] J. Logan, R. Harder, L. Li, D. Haskel, P. Chen, R. Winarski, P. Fuesz, D. Schlagel, D. Vine, C. Benson, and I. McNulty, Hard X-ray polarizer to enable simultaneous threedimensional nanoscale imaging of magnetic structure and lattice strain, J. Synchrotron Radiat. 23, 1210 (2016).

[22] M. Suzuki, Hard-X-ray magnetic microscopy and local magnetization analysis using synchrotron radiation, Microscopy 63, i14.1 (2014).

[23] B. Shen, Z. Bu, J. Xu, T. Xu, L. Ji, R. Li, and Z. Xu, Exploring vacuum birefringence based on a $100 \mathrm{PW}$ laser and an X-ray free electron laser beam, Plasma Phys. Controlled Fusion 60, 044002 (2018).

[24] A. B. Temnykh, Delta undulator for Cornell energy recovery linac, Phys. Rev. Accel. Beams 11, 120702 (2008).

[25] S. Sasaki, Analyses for a planar variably-polarizing undulator, Nucl. Instrum. Methods Phys. Res., Sect. A 347, 83 (1994).

[26] C. Spezzani, E. Allaria, M. Coreno, B. Diviacco, E. Ferrari, G. Geloni, E. Karantzoulis, B. Mahieu, M. Vento, and G. De Ninno, Coherent Light with Tunable Polarization from Single-Pass Free-Electron Lasers, Phys. Rev. Lett. 107, 084801 (2011).

[27] E. Allaria et al., Control of the Polarization of a VacuumUltraviolet, High-Gain, Free-Electron Laser, Phys. Rev. X 4, 041040 (2014).

[28] K. J. Kim, A synchrotron radiation source with arbitrarily adjustable elliptical polarization, Nucl. Instrum. Methods Phys. Res. 219, 425 (1984).

[29] K. J. Kim, Circular polarization with crossed-planar undulators in high-gain FELs, Nucl. Instrum. Methods Phys. Res., Sect. A 445, 329 (2000).

[30] H. Deng, T. Zhang, L. Feng, C. Feng, B. Liu, X. Wang, T. Lan, G. Wang, W. Zhang, X. Liu, J. Chen, M. Zhang, G. Lin, M. Zhang, D. Wang, and Z. Zhao, Polarization switching demonstration using crossed-planar undulators in a seeded free-electron laser, Phys. Rev. Accel. Beams 17, 020704 (2014).

[31] Y. Ding and Z. Huang, Statistical analysis of crossed undulator for polarization control in a self-amplified spontaneous emission free electron laser, Phys. Rev. Accel. Beams 11, 030702 (2008).

[32] K. J. Kim, Y. Shvyd'ko, and S. Reiche, A Proposal for an $\mathrm{X}$-Ray Free-Electron Laser Oscillator with an EnergyRecovery Linac, Phys. Rev. Lett. 100, 244802 (2008).

[33] K. Li and H. Deng, Gain-guided X-ray free-electron laser oscillator, Appl. Phys. Lett. 113, 061106 (2018).

[34] T. Wang et al., Femtosecond Single-Shot Imaging of Nanoscale Ferromagnetic Order in $\mathrm{Co} / \mathrm{Pd}$ Multilayers Using Resonant X-Ray Holography, Phys. Rev. Lett. 108, 267403 (2012).

[35] K.-J. Kim, Z. Huang, and R. Lindberg, Synchrotron Radiation and Free-Electron Lasers: Principles of Coherent X-ray Generation (Cambridge University Press, Cambridge, England, 2017), https://doi.org/10.1017/ 9781316677377.

[36] K. Li, M. Song, and H. Deng, Simplified model for fast optimization of a free-electron laser oscillator, Phys. Rev. Accel. Beams 20, 030702 (2017). 
[37] N. M. Kroll, P. L. Morton, and M. N. Rosenbluth, Freeelectron lasers with variable parameter wigglers, IEEE J. Quantum Electron. 17, 1436 (1981).

[38] C. Emma, N. Sudar, P. Musumeci, A. Urbanowicz, and C. Pellegrini, High efficiency tapered free-electron lasers with a prebunched electron beam, Phys. Rev. Accel. Beams 20, 110701 (2017).

[39] D. Prosnitz, A. Szoke, and V. K. Neil, High-gain, freeelectron laser amplifiers: Design considerations and simulation, Phys. Rev. A 24, 1436 (1981).

[40] A. Mak, F. Curbis, and S. Werin, Phase jump method for efficiency enhancement in free-electron lasers, Phys. Rev. Accel. Beams 20, 060703 (2017).

[41] A. Mak, F. Curbis, and S. Werin, Model-based optimization of tapered free-electron lasers, Phys. Rev. Accel. Beams 18, 040702 (2015).

[42] M. Born, E. Wolf, A. B. Bhatia, P. C. Clemmow, D. Gabor, A. R. Stokes, A. M. Taylor, P. A. Wayman, and W. L. Wilcock, Princ. Opt., 7th ed. (Cambridge University Press, Cambridge, England, 1999), https://doi.org/ 10.1017/CBO9781139644181.

[43] S. Reiche, GENESIS 1.3: a fully 3D time-dependent FEL simulation code, Nucl. Instrum. Methods Phys. Res., Sect. A 429, 243 (1999).
[44] J. G. Karssenberg, P. J. Van Der Slot, I. V. Volokhine, J. W. Verschuur, and K.J. Boller, Modeling paraxial wave propagation in free-electron laser oscillators, J. Appl. Phys. 100, 093106 (2006).

[45] N. S. Huang, K. Li, and H. X. Deng, BRIGHT: the threedimensional X-ray crystal Bragg diffraction code, Nucl. Sci. Tech. 30, 39 (2019).

[46] R. R. Lindberg, K. J. Kim, Y. Shvyd'Ko, and W. M. Fawley, Performance of the X-ray free-electron laser oscillator with crystal cavity, Phys. Rev. Accel. Beams 14, 010701 (2011).

[47] T. Ishikawa, X-ray monochromators for circularly polarized incident radiation, Rev. Sci. Instrum. 60, 2058 (1989).

[48] C. Malgrange, C. Carvalho, L. Braicovich, and J. Goulon, Transfer of circular polarization in Bragg crystal X-ray monochromators, Nucl. Instrum. Methods Phys. Res., Sect. A 308, 390 (1991).

[49] Y. Shvyd'ko, Output coupling from x-ray free-electron laser cavities with intracavity beam splitters, Phys. Rev. Accel. Beams 22, 100703 (2019).

[50] Y. Shvyd'Ko and R. Lindberg, Spatiotemporal response of crystals in X-ray Bragg diffraction, Phys. Rev. Accel. Beams 15, 100702 (2012). 